\title{
EVALUACIÓN Y SELECCIÓN DEL LAYOUT DE UNA INSTALACIÓN CON EL EMPLEO DE UN ENFOQUE HÍBRIDO SIMULACIÓN MULTIATRIBUTO
}

\section{EVALUATION AND SELECTION OF THE LAYOUT OF AN INSTALLATION WITH THE USE OF A HYBRID APPROACH MULTIATRIBUTE SIMULATION}

\author{
Rosario Garza Rios \\ Universidad Tecnológica de La Habana José Antonio \\ Echeverría - CUJAE Facultad de Ingeniería Industrial \\ La Habana, Cuba \\ rosariog@ind.cujae.edu.cu
}

\author{
Edith Martinez Delgado \\ Universidad Tecnológica de La Habana José Antonio \\ Echeverría - CUJAE, Facultad de Ingeniería Industrial \\ La Habana, Cuba \\ edithmd@ind.cujae.edu.cu
}

Fecha de recepción: 02/12/2018- Fecha de aprobación:02/04/2019

\section{RESUMEN}

El desempeño exitoso de las organizaciones de producción de bienes materiales o servicio pasan necesariamente por una correcta localización de los elementos que conforman el sistema productivo, de modo que permita responder satisfactoriamente no sólo a las situaciones actuales sino también a posibles escenarios futuros. Entre las técnicas de la Investigación de Operaciones, que resultan de utilidad para analizar el comportamiento de los sistemas, está la Simulación, la que ha demostrado sus ventajas en la cuantificación de variables de salida o indicadores de interés, asociados a cada escenario diseñado. En particular, en este artículo se evidencia la conveniencia del uso de la Simulación para evaluar el layout de una organización ante distintos escenarios y múltiples criterios. Se muestran los pasos de un enfoque híbrido basado en la Simulación y técnicas Multicriterio y su aplicación a dos casos de estudio. Para evaluar el comportamiento de los sistemas según el layout diseñado, se generan y resuelven respectivos modelos de Simulación a través del lenguaje ARENA en su versión 15.0. Además, como técnicas multiatributo para la selección del layout preferido se aplican los métodos: lexicográfico y EDIPO; en este último caso con el uso del software DECISION.

PALABRAS CLAVE: Distribución en Planta; Simulación; Técnicas Multicriterio; Enfoque Híbrido.

\section{ABSTRACT}

The successful performance of the production organizations of material goods or service necessarily go through a correct location of the elements that make up the productive system,

\footnotetext{
"Visión de Futuro" Año 16, Volumen N²3 N², Julio - Diciembre 2019 - Pág. 294 - 311

URL de la Revista: http://visiondefuturo.fce.unam.edu.ar/index.php/visiondefuturo/index

URL del Documento: http://visiondefuturo.fce.unam.edu.ar/index.php/visiondefuturo/issue/view/

ISSN 1668 - 8708 - Versión en Línea

E-mail: revistacientifica@fce.unam.edu.ar
}

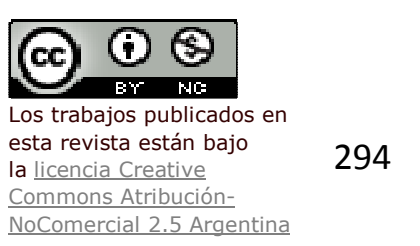


so that it can satisfactorily respond not only to current situations but also to possible future scenarios. Among the techniques of Operations Research, which are useful for analyzing the behavior of systems, there is Simulation, which has demonstrated its advantages in the quantification of output variables or indicators of interest, associated with each designed scenario. In particular, this article demonstrates the convenience of using Simulation to evaluate the layout of an organization in different scenarios and multiple criteria. The steps of a hybrid approach based on Simulation and Multicriteria techniques and their application to two case studies are shown. To evaluate the behavior of the systems according to the designed layout, respective models of Simulation are generated and solved through the ARENA language in its version 15.0. In addition, as multi-attribute techniques for the selection of the preferred layout, the following methods are applied: lexicographic and EDIPO; in the latter case with the use of the DECISION software.

KEY WORDS: Layout; Simulation; Multicriteria techniques; Hybrid Approach.

\section{INTRODUCCIÓN}

El rápido incremento de las demandas de productos exige de las industrias, una mayor capacidad para elevar su ritmo de producción y al mismo tiempo hacer que los procesos sean más eficientes disminuyendo el costo con una alta efectividad.

La conformación de una distribución en planta acorde con los objetivos y requerimientos de las organizaciones constituye una reserva potencial para mejorar la productividad. Estudiosos estiman que del 20 al 50\% de los gastos totales de operación en que se incurren dentro del área de fabricación, se pueden atribuir a la disposición de la planta, y que un layout eficiente reduce probablemente esos costos entre el 10 y el 30\% (Chase y Jacob, 2013), (Marcoux et al, 2005). Se puede agregar entonces que la distribución en planta es una de las tareas más significativas y una de las más críticas para mejorar la tasa de productividad y lograr mejorar el flujo de trabajo en la ruta de producción y/o servicio.

Diversos son los procedimientos desarrollados para realizar un diseño en planta eficiente (Panwar y Patra, 2017), (Wiendahl, et al, 2015), sin embargo, es escaso el uso de herramientas cuantitativas para evaluar las alternativas generadas del layout y así comprobar la efectividad del comportamiento de los sistemas productivos (de bienes materiales y de servicios) que previamente sean diseñados. Autores como Mejía y Galofre (2008), Rodríguez, et al, (2002), Suárez (2017), Pochamarn et al, (2008), Acuña et al, (2002), reconocen la utilidad de los modelos de Simulación de eventos discretos en la distribución en planta y expresan la necesidad de considerar el carácter estocástico de los problemas en el mundo real.

\footnotetext{
"Visión de Futuro" Año 16, Volumen N 23 N², Julio - Diciembre 2019 - Pág. 294 - 311

URL de la Revista: http://visiondefuturo.fce.unam.edu.ar/index.php/visiondefuturo/index

URL del Documento: http://visiondefuturo.fce.unam.edu.ar/index.php/visiondefuturo/issue/view/16

ISSN 1668 - 8708 - Versión en Línea 
El empleo de la Simulación Discreta con el apoyo del lenguaje de Simulación ARENA permite evaluar el diseño de layout para darle solución a una situación dada. Esta técnica matemática hace posible predecir el comportamiento de sistemas de manufactura o servicio, mediante la observación de los movimientos o la interacción de los elementos del sistema. Entre las ventajas que posee la Simulación que la hacen aplicable para evaluar el diseño de la distribución en planta se encuentran:

- Probar la factibilidad del sistema propuesto.

- No es necesario llegar a la interrupción de operaciones en la compañía, resultando ser más económico imitar el sistema.

- Suministra información del comportamiento del sistema ante distintos escenarios sobre los cuales se puede explorar.

- Permite proporcionar a los empleados y directivos información acerca de los nuevos sistemas propuestos.

Si bien ya ha quedado claro la evidente ventaja que constituye la aplicación de la Simulación como herramienta para la evaluación de las alternativas de layout, hay que tener en cuenta que sólo con dicha evaluación no es suficiente para tomar la decisión de cuál distribución en planta implementar si se desean considerar múltiples variables o atributos donde algunos valores son obtenidos a través de la Simulación, mientras que otros están disponibles con la propia distribución realizada.

Diversas son las situaciones prácticas cuyas decisiones dependen de más de una medida de efectividad por lo que un enfoque monocriterio resulta insuficiente para llegar a una solución, de esta forma se puede hablar de la existencia de un paradigma multicriterial donde deben resolverse una serie de trade-offs que incluyen criterios en conflicto y se abarcan tanto problemas de toma de decisiones con múltiples objetivos como con múltiples atributos, estos últimos también conocidos como problema de decisión multicriterio en espacios discretos. En el desarrollo de la Teoría de la Decisión se reconocen dos escuelas: una normativa y otra descriptiva.

La primera, desarrollada fundamentalmente por Estados Unidos e Inglaterra, se basa en prescribir normas, de modo que el decisor piense sistemáticamente; lo ayuda a conocer la coherencia de sus decisiones con sus preferencias. Esta corriente tiene un amplio fundamento matemático en la modelación del problema, el conjunto de axiomas definidos, etc., y utiliza como modelo, la racionalidad. Para resolver problemas de decisión Multicriterio en espacios discretos ha desarrollado la construcción de una función de valor multiatributo. Por otra parte, la escuela descriptiva desarrollada por Europa Continental (Francia, Holanda 
y Bélgica), renuncia a la idea de lo racional y trata de hacer un reflejo del modo en que el decisor toma las decisiones, lo ayuda modelando su comportamiento tal y como es. Aunque menos impresionante que la escuela normativa, también posee una formulación matemática.

Múltiples son los métodos desarrollados para resolver un problema multiatributo (Fernández y Escribano, 2012), (Lara, 2016), (Saaty y Vargas, 2012), (Soto de la Vega et al, 2014), (Aragonés, 2010), (Berumeny y Llamazares, 2007), aunque no hay evidencia de que alguno sea el mejor. Incluso existen diferentes artículos donde se realiza una comparación entre algunos de los métodos, llegándose a la conclusión de que se obtiene la misma solución referente a la alternativa seleccionada (Garza, et al, 2012), (Tavella et al, 2014). (Fernández y Escribano, 2002). La elección del método empleado se fundamenta por la información disponible y el objetivo que se persigue con su aplicación.

Podría entonces enunciarse como problema a resolver el siguiente: ¿Cómo obtener el layout que de forma más satisfactoria considere el carácter estocástico y contradictorio de los elementos del sistema productivo que lo conforman?

Para decidir cuál es la mejor distribución y dar respuesta al problema planteado, las autoras realizan el siguiente planteamiento: la utilización de un enfoque híbrido Simulación y técnicas de decisión multicriterio en espacios discretos (multiatributo), permitirá, teniendo en cuenta los intereses de los decisores, seleccionar cuál de los diseños realizados cumple mejor con los atributos definidos, constituyendo este planteamiento la hipótesis.

El presente artículo tiene como objetivo mostrar la aplicación de la Simulación y un método multiatributo para evaluar y seleccionar el layout más conveniente, según preferencias del decisor. Para ello se utilizan dos casos de estudio, uno referido a una instalación de producción de bebidas y otro de servicio gastronómico.

\section{DESARROLLO}

El empleo de herramientas cuantitativas para la evaluación y selección del layout constituye un aspecto esencial en el desarrollo del presente trabajo.

A continuación se presenta el conjunto de pasos que se desarrollan en un enfoque híbrido con el empleo de la Simulación y técnicas multicriterio. Para mayor detalle se puede consultar el procedimiento de González, et al, (2014). Dichos pasos son:

1. Planteamiento de la situación a resolver.

2. Generación de las alternativas de decisión.

3. Modelo de simulación a través del ARENA 15.0

4. Obtención de resultados.

5. Aplicación de una técnica multiatributo para la obtención de la mejor alternativa de decisión.

\footnotetext{
"Visión de Futuro" Año 16, Volumen N²3 N², Julio - Diciembre 2019 - Pág. 294 - 311

URL de la Revista: http://visiondefuturo.fce.unam.edu.ar/index.php/visiondefuturo/index

URL del Documento: http://visiondefuturo.fce.unam.edu.ar/index.php/visiondefuturo/issue/view/16

ISSN 1668 - 8708 - Versión en Línea 
En la exposición de ambos casos de estudio se parte del planteamiento del problema. En éste se considera una breve descripción cualitativa de la situación de decisión que será objeto de análisis, así como la información fundamental recopilada que caracterizan al sistema, identificando cuáles son las variables que responden a un comportamiento aleatorio y cuál es la distribución probabilística que sigue cada una. Posteriormente se hace alusión a las alternativas de decisión, que en este caso son los layout que han sido generados. Se muestran las posibilidades del uso de la Simulación Discreta para evaluar la efectividad de los diseños de distribución en planta en dos casos de estudio, los cuales presentan características diferentes. Otro aspecto de interés que se ofrece, es la aplicación de técnicas de apoyo a la toma de decisiones multicriterio las que permitirán seleccionar el mejor layout considerando los resultados arrojados por la simulación y los intereses de los decisores.

\section{Caso de estudio 1: Instalación productiva}

Este caso de estudio hace referencia a una instalación de embotellamiento y envasado de bebidas. La misma dispone de la maquinaria adecuada para la producción de cuatro tipos de bebidas (dos marcas de cerveza y dos sabores de refrescos). Para ello cuenta con los siguientes equipos: 4 embotelladoras, 5 máquinas envasadoras (2 para botellas de $350 \mathrm{ml}$ y 3 para pomos plásticos de 1,5 litro) y 3 cintas de transporte.

El proceso de producción consiste en la entrada de las materias primas (pomos y botellas) a través de las cintas transportadoras a las embotelladoras que se encargan automáticamente del trabajo y se realiza un control de calidad. Una vez embotelladas, las botellas y pomos pasan a través de la cinta transportadora al puesto de envase donde se conforman paquetes de 6 botellas de cerveza y paquetes de 9 pomos de refresco en dicho puesto. Finalmente son almacenados para su posterior distribución a las distintas entidades comerciales. En la figura № 1 se muestra de forma resumida cómo se comporta el flujo de trabajo de la embotelladora.

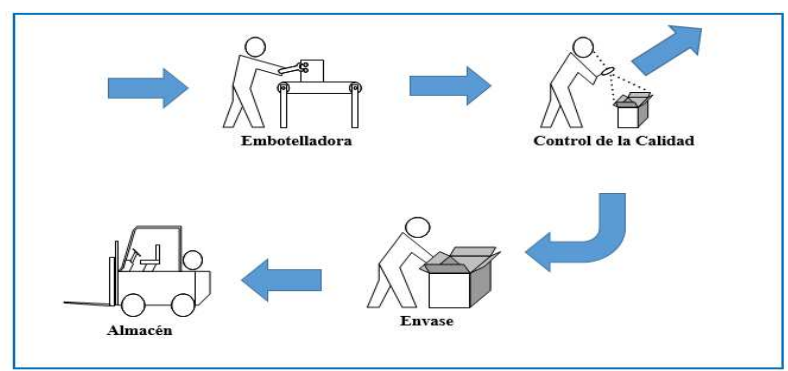

Figura $\mathrm{N}^{\circ}$ 1. Flujo de trabajo en la instalación productiva Fuente: Elaboración Propia

\footnotetext{
"Visión de Futuro" Año 16, Volumen N²3 N², Julio - Diciembre 2019 - Pág. 294 - 311

URL de la Revista: http://visiondefuturo.fce.unam.edu.ar/index.php/visiondefuturo/index

URL del Documento: http://visiondefuturo.fce.unam.edu.ar/index.php/visiondefuturo/issue/view/16

ISSN 1668 - 8708 - Versión en Línea 
El diseño actual de la planta provoca pérdidas de material y retrocesos que hacen excesivo el tiempo de ciclo, por lo que se desea rediseñar la planta con el objetivo de obtener mejores resultados en este sentido.

Utilizando el Systematic Layout Planning (SLP) se obtuvieron distintas alternativas de nuevos diseños de la planta embotelladora de cervezas y refresco, presentándose aquellas dos de mayor interés, que se describen a continuación.

\section{Alternativa 1}

El diseño de esta alternativa está dado fundamentalmente por la secuencia de operaciones que requiere la producción de bebidas, que sería almacén-embotelladoraenvasadora-almacén, así como la forma de la instalación, por lo que se propone utilizar una distribución en $U$. Otras consideraciones consisten en reservar ciertos espacios y requerimientos según se especifica a continuación:

- Un área de la instalación para el almacén de $50 \mathrm{~m}^{2}$ (tanto para materias primas y materiales como para la producción terminada).

- Dos zonas: una dedicada al comedor $\left(23 \mathrm{~m}^{2}\right)$ y otra a las oficinas $\left(20 \mathrm{~m}^{2}\right)$.

- Se requiere que el almacén esté de forma contigua a la zona que se utilizará para la circulación de los camiones que se encargarán del transporte de productos y materiales por lo que ésta deberá tener acceso a la vía y tener espacio suficiente para permitir la circulación de hasta 3 camiones de dimensiones $3 \times 2 \times 3 \mathrm{~m}$.

- La Dirección ha establecido que desde las oficinas se puedan controlar las actividades del almacén y de producción.

Esta alternativa intenta explotar al máximo los recursos disponibles. En la Figura $\mathrm{N}^{\circ} 2$ se muestra la distribución obtenida de la alternativa 1.

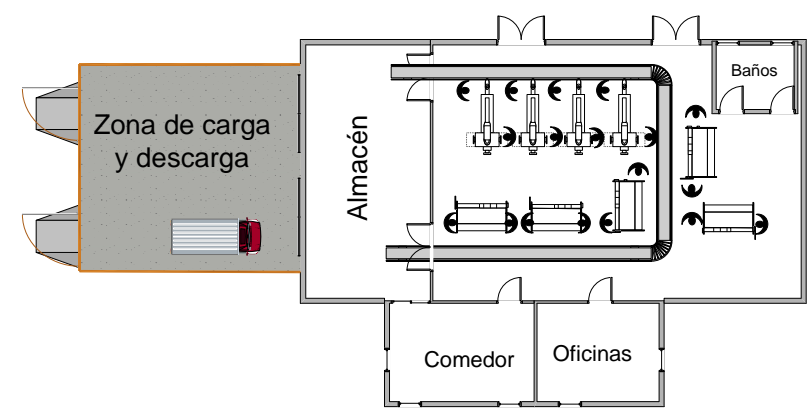

Figura $N^{\circ}$ 2. Distribución de las maquinas en el área de producción para la alternativa 1

Fuente: Elaboración Propia 


\section{Alternativa 2}

El diseño de la alternativa 2 se elaboró considerando un nuevo enfoque que tiene como objetivo la optimización del ciclo productivo reduciendo los tiempos de transporte entre los puestos de trabajo, ya que ésta trata de aprovechar al máximo el espacio ocupado. Con esta variante se elimina el tiempo de transporte entre los puestos de trabajo al asignar una envasadora a cada una de las maquinas embotelladoras, principal diferencia con la alternativa 1. Los demás requerimientos especificados se mantienen. En la Figura $\mathrm{N}^{\circ} 3$ se muestra el diseño de dicha alternativa.

Una vez generadas las alternativas de mayor utilidad, se procede a la definición de variables y atributos de interés para evaluar la efectividad de las mismas. En esta aplicación (Caso de estudio 1) se determinaron las variables o criterios cuantitativos y cualitativos que incidirán en la decisión final, siendo éstas las siguientes:

- Cantidad de paquetes de productos producidos (paquetes/día).

- Tiempos muertos (minutos/día).

- Ciclo productivo (minutos).

- Utilización de los recursos (\%).

- Cantidad total de recursos empleados.

- Preferencias de cercanía entre las diferentes zonas de la instalación.

- Retrocesos (orden no lineal de la secuencia que sigue el producto a través de la instalación productiva).

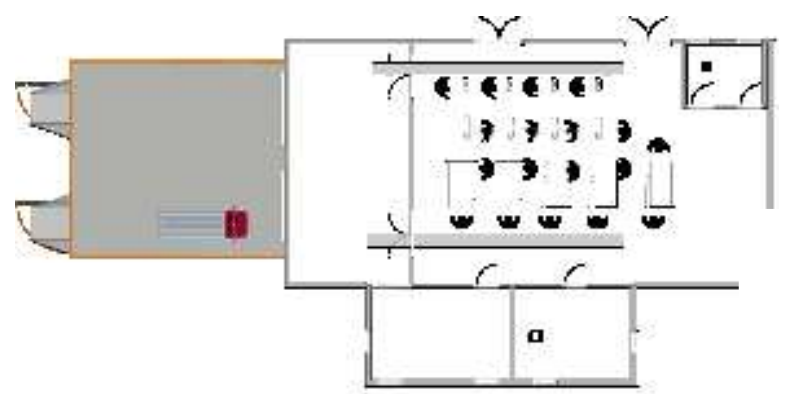

Figura № 3. Distribución de las máquinas en el área de producción para alternativa 2

Fuente: Elaboración Propia

Los valores de las variables cuantitativas se obtienen a través de la Simulación Discreta, específicamente utilizando el lenguaje ARENA versión 15.0, mientras que las variables cualitativas se consideran y obtienen directamente del layout. Para las dos últimas variables se utiliza un patrón de evaluación cuya escala oscila entre el 0 (resultado más favorable) y el 1 (resultado más desfavorable).

"Visión de Futuro" Año 16, Volumen No 23 N², Julio - Diciembre 2019 - Pág. 294 - 311

URL de la Revista: http://visiondefuturo.fce.unam.edu.ar/index.php/visiondefuturo/index

URL del Documento: http://visiondefuturo.fce.unam.edu.ar/index.php/visiondefuturo/issue/view/16

ISSN 1668 - 8708 - Versión en Línea 
Resulta de utilidad animar el comportamiento del sistema que se estudia, aprovechando la facilidad del lenguaje ARENA (Kelton et al, 2009) en las figuras № 4 y No 5 aparecen los modelos de Simulación animados de las dos alternativas diseñadas.

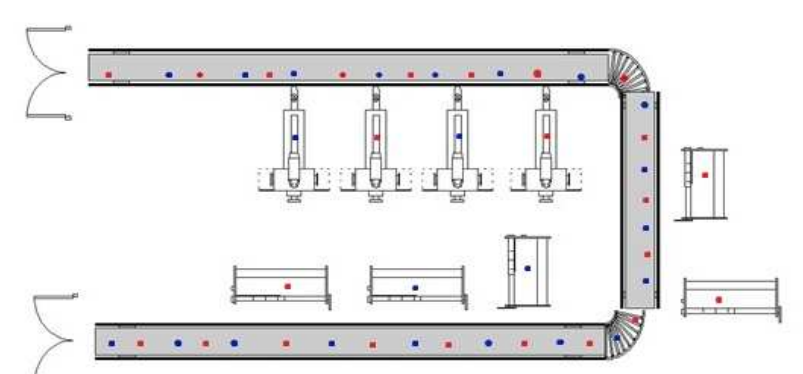

Figura № 4. Modelo de simulación para la alternativa 1 Fuente: Elaboración Propia

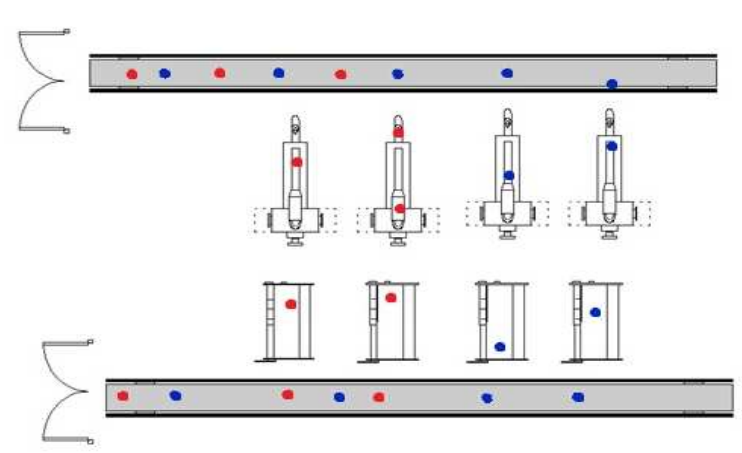

Figura $N^{\circ}$. Modelo de simulación alternativa 2 Fuente: Elaboración Propia

Con el propósito de garantizar la fiabilidad de los resultados obtenidos a través de la Simulación, es necesario realizar un diseño de experimentos de ésta (Kelton et al, 2009) (Martínez et al, 2018); el que consiste en determinar el número de réplicas y la longitud de las mismas. La longitud de las corridas está en correspondencia con las características de la instalación productiva, en este caso es un sistema con término y está fijada por el régimen de trabajo que es de 12 horas de jornada laboral. Para determinar el número de réplicas se define como variable independiente a utilizar el tiempo medio de permanencia en el sistema (o sea el tiempo que demora la producción de un lote).

Para el cálculo del número de réplicas necesarias $(n)$ de la variable seleccionada se utiliza la ec. 1, (Kelton, et al, 2009):

$$
n=n_{0}\left(\frac{H_{r}}{H_{d}}\right)^{2}
$$

Donde:

$\mathrm{n}_{0}$ : número de réplicas piloto.

$\mathrm{H}_{\mathrm{r}}$ : es la semi-amplitud del intervalo (half-width) en la salida de la muestra piloto.

$\mathrm{H}_{\mathrm{d}}$ : es la semi-amplitud del intervalo deseada.

En la tabla $\mathrm{N}^{0} 1$ se muestran los resultados obtenidos para las dos alternativas. 
Tabla № 1. Número de réplicas para cada modelo de Simulación

\begin{tabular}{|c|c|c|}
\hline & & \\
& Alternativa 1 & Alternativa 2 \\
\hline $\mathrm{N}_{0}$ & 5 & 5 \\
\hline $\mathrm{H}_{\mathrm{r}}$ & 16,9935 & 21,936 \\
\hline $\mathrm{H}_{\mathrm{d}}$ & 4 & 4 \\
\hline $\mathrm{n}$ & 91 & 151 \\
\hline
\end{tabular}

Fuente: Elaboración propia

Como se puede apreciar en la tabla, la longitud de corrida queda dada por 91 y 151 corridas para las alternativas 1 y 2 respectivamente.

Un resumen comparativo de los resultados de la Simulación de las alternativas se muestra en la Tabla $N^{\circ} 2$, incluyéndose la variación favorable $\left(\Delta_{1}\right.$ y $\left.\Delta_{2}\right)$ obtenida en las alternativas 1 y 2 respectivamente para cada criterio.

Tabla № 2. Resultados obtenidos en el Arena de las variables estudiadas

\begin{tabular}{|l|c|c|c|c|}
\hline \multicolumn{1}{|c|}{ Criterio } & \multicolumn{2}{c|}{ Alternativa } & \multirow{2}{*}{$\Delta_{1}$} & \multirow{2}{*}{$\Delta_{2}$} \\
\cline { 2 - 3 } & 1 & 2 & & \\
\hline Cantidad de paquetes de cervezas producidos & 118 & 113 & 5 & - \\
\hline Cantidad de paquetes de refresco producidos & 57 & 57 & 0 & 0 \\
\hline Tiempos muertos & 1,99 & 0,52 & - & 1,47 \\
\hline Ciclo productivo de la cerveza & 55,5 & 58,65 & 3,15 & - \\
\hline Ciclo productivo del refresco & 12,47 & 29,09 & 16,62 & - \\
\hline Utilización de las embotelladora & 89,96 & 86,85 & 3,11 & - \\
\hline Utilización de las envasadoras & 47,22 & 44,88 & 2,34 & - \\
\hline Cantidad total de recursos empleados & 9 & 8 & -1 & - \\
\hline Preferencias de cercanía & 0 & 0 & - & - \\
\hline Retrocesos & 0 & 1 & 1 & - \\
\hline
\end{tabular}

Fuente: Elaboración Propia

Considerando el carácter multicriterio de la decisión a tomar, se aplica el método lexicográfico (Lara, 2016). De los resultados obtenidos se puede apreciar que solamente en el criterio tiempos muertos, la alternativa 2 es mejor que la 1, por tanto se selecciona esta última como la mejor.

\section{Caso de estudio 2: Diseño de un restaurante de comida internacional y criolla}

En este caso se trata de un negocio que actualmente es una cafetería y se desea ampliar realizando reformas a la casa original, la cual dejará de ser utilizada como vivienda por sus ocupantes para que sea convertida en un restaurante/cafetería con las comodidades y prestaciones que permitan brindar un servicio de excelencia. Las especificaciones a tener en cuenta para el nuevo diseño son las que siguen:

\footnotetext{
“Visión de Futuro" Año 16, Volumen No 23 N², Julio - Diciembre 2019 - Pág. 294 - 311

URL de la Revista: http://visiondefuturo.fce.unam.edu.ar/index.php/visiondefuturo/index

URL del Documento: http://visiondefuturo.fce.unam.edu.ar/index.php/visiondefuturo/issue/view/16

ISSN 1668 - 8708 - Versión en Línea 
- Hacer uso de las zonas con que cuenta la casa. Éstas son:

$>$ Portal

$>$ Sala-Comedor

$>$ Estudio

$>$ Cocina

Garaje (en el subnivel)

$>$ Baño (piso superior)

$>$ Dormitorio (piso superior)

- Mantener la ubicación del baño y de la cocina, valorando introducir las mejoras que se requieran.

- Mantener el área de la cafetería actual, es decir en el garaje.

- Localizar una de las zonas de la casa para la creación de un almacén.

- Decidir otras zonas de atención al cliente incluyendo algún área para el despacho de bebidas, pudiendo considerar un ambiente climatizado en algunas de sus áreas.

El diseño del layout de la casa debe responder a los requerimientos del dueño de la propiedad, ampliando la capacidad de bridar el servicio gastronómico y con ello los ingresos.

Este caso se corresponde con un sistema de servicio de cola infinita (Hillier y Lieberman, 2015). Las variables de entrada siguen la distribución probabilística que se muestra en la Tabla № 3 .

Tabla № 3. Distribución probabilística de las variables de entrada

\begin{tabular}{|l|l|}
\hline Variable & Distribución \\
\hline Arribo de clientes (minutos) & $\mathrm{GAMM}(0,18-5,51)$ \\
\hline Tiempo para servir líquidos (minutos) & $1+1,56^{*} \operatorname{BETA}(2,19-2,1)$ \\
\hline Tiempo para preparar órdenes (horas) & $0,35+\operatorname{LOGN}(0,556-0,303)$ \\
\hline Tiempo de servir el pedido de la cocina (minutos) & $\operatorname{NORM}(1,44-0,365)$ \\
\hline Tiempo de consumo los clientes (minutos) & $\operatorname{TRIA}(2,11-1,17)$ \\
\hline Tiempo para pedido del postre (minutos) & $0,05+\operatorname{LOGN}(0,355-0,281)$ \\
\hline Tiempo para servir el postre (minutos) & $0,03+\operatorname{LOGN}(0,732-0,56)$ \\
\hline Tiempo para comer el postre (minutos) & $\operatorname{NORM}(5,98,1,45)$ \\
\hline Confección del vale (minutos) & $\mathrm{UNIF}(0,1,0,12)$ \\
\hline Tiempo de entrega de la cuenta (minutos) & $\operatorname{NORM}(0,691,0,178)$ \\
\hline Cálculo del vuelto (minutos) & $\mathrm{UNIF}(0,1-0,15)$ \\
\hline Entrega del vuelto (minutos) & $\operatorname{NORM}(0,974-0,208)$ \\
\hline Consumo promedio (pesos cubanos convertibles o CUC) & 27 \\
\hline
\end{tabular}

Fuente: Elaboración Propia

Con el empleo del método SLP se generaron dos alternativas de diseño del nuevo restaurante. A continuación se expone en qué consiste cada alternativa propuesta, así como la distribución realizada. 


\section{Alternativa 1}

La distribución de las distintas áreas de la casa quedan de la manera que sigue: Un salón climatizado, que ocupará la sala y el estudio original de la casa, en la que se colocarán 5 mesas: 2 para 4 personas y 3 para 2 personas, lo más cercanas posible a la cocina sin afectar la circulación del personal ni de los clientes. Se incorporará una barra para el despacho de bebidas a los clientes, donde se encontrará la caja de pago para efectuar el cierre de las cuentas.

En el portal se encontrará la zona al aire libre con una mesa de 4 personas y una para 2 personas, esta zona estará destinada para aquellos clientes que prefieran el fresco del ambiente o para aquellos clientes fumadores.

El garaje se mantendrá en su sitio con cambios menores en su estética y una renovación de pintura funcionando para atender a los clientes que prefieran disfrutar de una comida rápida o solamente para recoger un encargo.

A la cocina se le incorporarán sendas esclusas para las órdenes y para los platos sucios que estarán ubicadas con salida al salón en la zona donde antes estaba el estudio.

En el segundo piso el baño mantendrá su ubicación, solamente eliminando la bañera y ubicando una nueva repisa con lavamanos y el excusado de forma tal que permita la mayor movilidad al cliente. El almacén estará ubicado en el antiguo dormitorio y contará con una puerta que de acceso directo a las escaleras para facilitar la rapidez del tránsito de empleados.

En la figura № 6 se muestra el layout de la alternativa 1 (descrita anteriormente).

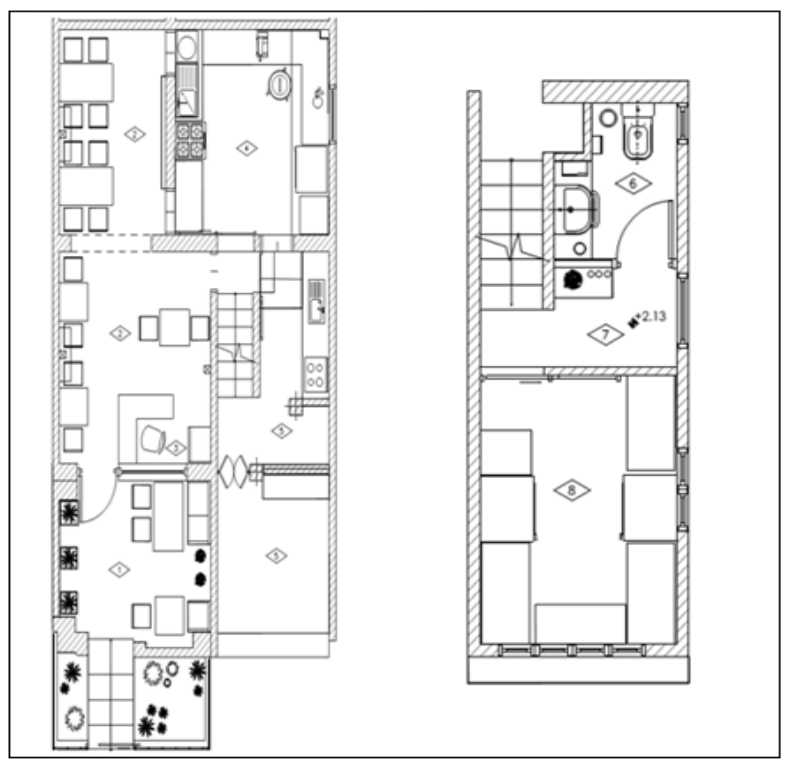

Figura $N^{\circ} 6$. Vista en planta de la alternativa 1 Fuente: Elaboración Propia

\footnotetext{
"Visión de Futuro" Año 16, Volumen Nº 23 N², Julio - Diciembre 2019 - Pág. 294 - 311

URL de la Revista: http://visiondefuturo.fce.unam.edu.ar/index.php/visiondefuturo/index

URL del Documento: http://visiondefuturo.fce.unam.edu.ar/index.php/visiondefuturo/issue/view/16

ISSN 1668 - 8708 - Versión en Línea 


\section{Leyenda}

1. Zona de atención en el portal (al aire libre), 2. Zona de atención principal (salón climatizado), 3.Barra, 4. Cocina, 5.Garaje, 6.Baño, 7.Pasillo, 8.Almacén.

\section{Alternativa 2}

En esta alternativa se plantea utilizar el espacio del estudio original de la casa como almacén, designar una zona para reservado o VIP proponiéndose el dormitorio original que se encuentra en el segundo piso de la casa, manteniéndose las restantes áreas como se expusieron en la alternativa 1 . En la figura $\mathrm{N}^{0} 7$ se muestra el diseño de dicha alternativa.

Siguiendo los pasos para la evaluación de las variantes generadas se definieron como atributos a valorar los siguientes:

- Cantidad de clientes satisfechos (reciben el servicio).

- Cantidad de clientes que abandonan.

- Tiempo medio de estancia en el restaurante (horas).

- Tiempo medio de espera (minutos).

- Utilización de los trabajadores (\%).

- Ingreso medio (CUC/día).

- Requerimientos de seguridad y salud de los comensales (existen o no esclusas para la entrada-salida de platos limpios y sucios).

- Requerimientos de seguridad y salud de los empleados (riesgo de caída).

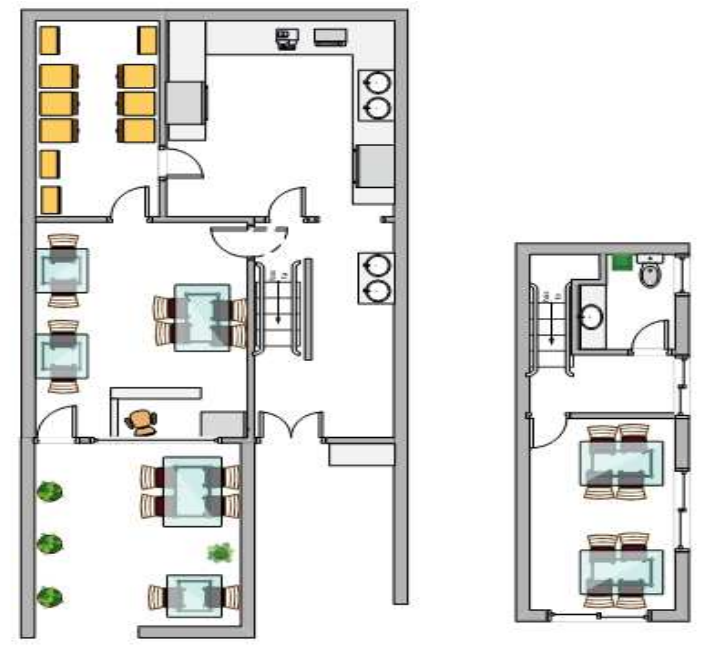

Figura $N^{\circ} 7$. Vista en planta de la alternativa 2

Fuente: Elaboración Propia

Una vez definidas las variables, se modelaron las dos alternativas diseñadas para el nuevo restaurante a través del ARENA 15.0. Utilizando una muestra piloto de 5 corridas se determinó la cantidad de réplicas necesarias para poder inferir a través de los resultados de 
la Simulación, con la aplicación de la expresión 1, determinándose que con estas 5 réplicas se garantiza la fiabilidad de los resultados al obtenerse un tamaño óptimo de réplica de 3,23 y 4,17 que se aproximan a 4 y 5 réplicas para las alternativas 1 y 2 respectivamente. En lo que respecta a la longitud de las corridas, queda determinada por la jornada de atención del nuevo restaurante y es de 10 horas por día.

Entre los criterios que resultan de interés para satisfacer a los comensales está el tiempo medio de espera. En las figuras $\mathrm{N}^{\circ} 8$ y $\mathrm{N}^{\circ} 9$ se muestra el ploteo de esta variable por comensal y para las alternativas 1 y 2 respectivamente. Se puede apreciar el comportamiento de este tiempo tanto durante el estado transitorio del sistema con un comportamiento similar en ambas alternativas (creciendo) y en el estado estacionario donde el tiempo de espera para ser servido se mantiene en un rango aproximado de 28 a 40 minutos para la alternativa 1 y entre 30 y 44 minutos para la alternativa 2 .

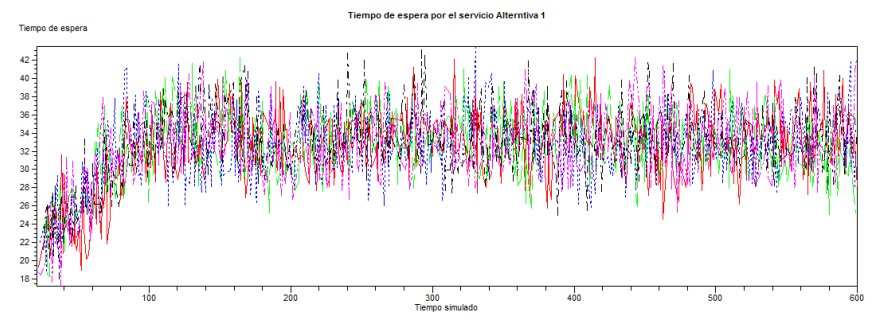

Figura $N^{\circ}$ 8. Tiempo medio de espera por el servicio en Alternativa 1

Fuente: Elaboración Propia

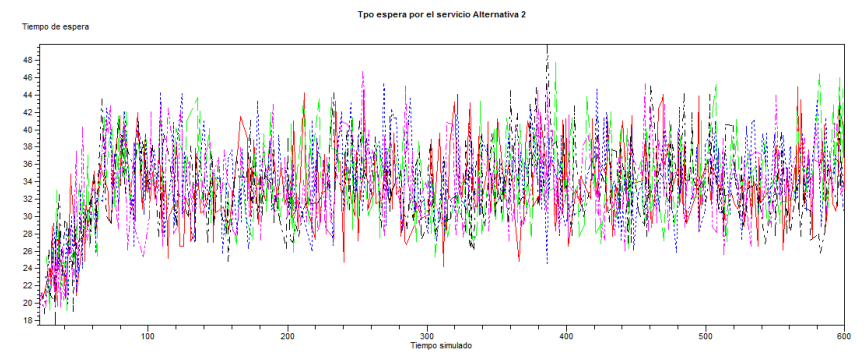

Figura NN$^{\circ}$ 9. Tiempo medio de espera por el servicio en Alternativa 2

Fuente: Elaboración Propia

En la Tabla $N^{\circ} 4$ se muestran los valores de los criterios cuantitativos seleccionados para la toma de decisiones para cada alternativa de diseño generada. Las mismas fueron obtenidas a través de las variables propias de la simulación con ARENA.

\footnotetext{
“Visión de Futuro" Año 16, Volumen No 23 N², Julio - Diciembre 2019 - Pág. 294 - 311

URL de la Revista: http://visiondefuturo.fce.unam.edu.ar/index.php/visiondefuturo/index

URL del Documento: http://visiondefuturo.fce.unam.edu.ar/index.php/visiondefuturo/issue/view/16

ISSN 1668 - 8708 - Versión en Línea 
Tabla № 4. Resultados para la comparación de las alternativas

\begin{tabular}{|l|c|c|}
\hline \multirow{2}{*}{ Criterio } & \multicolumn{2}{c|}{ Alternativas } \\
\cline { 2 - 3 } & 1 & 2 \\
\hline Clientes satisfechos & 395 & 345 \\
\hline Clientes perdidos & 146 & 212 \\
\hline Tiempo medio de estancia (h) & 1,53 & 1,49 \\
\hline Tiempo medio de espera (min) & 32,588 & 34,0778 \\
\hline Utilización de los obreros & 96,237 & 97,115 \\
\hline Ingreso (CUC/ día) & 4008,2 & 3352,8 \\
\hline
\end{tabular}

Fuente: Elaboración Propia

Como se puede apreciar (Tabla $N^{\circ} 3$ ) las evaluacione s de los atributos o criterios que se obtienen para cada alternativa favorecen (en cuatro de los cinco criterios) a la alternativa 1; es decir, la elección no está determinada por la existencia de una alternativa dominante, por lo que se debe aplicar algún método multiatributo de los múltiples existentes. En este caso, la elección de la alternativa 1 se puede confirmar con la aplicación del método multiatributo EDIPO, implementado en el software DECISION. EI mismo se basa en la filosofía del ELECTRE III y el empleo de una función de preferencia fuzzy. La ponderación de los criterios puede introducirse directamente en el software o ser obtenida resolviendo la ecuación de indiferencia de forma interactiva con el decisor. En cualquier caso los coeficientes de ponderación de los criterios quedan normalizados (Fernández, 1999), (Fernández, et al, 2010).

En la Tabla $N^{\circ} 5$ se muestra la matriz de decisión utilizada en el sistema informático DECISION empleado y en la figura $\mathrm{N}^{\circ} 10$ se muestran algunas pantallas del mismo; en particular, aparece primeramente la matriz de decisión, una vez que se han resuelto las ecuaciones de indiferencia para obtener la ponderación de cada uno de los criterios considerados y posteriormente se presenta el ordenamiento de las alternativas con el valor asociado de la función de utilidad.

Tabla № 5 . Matriz de decisión y criterios ponderados

\begin{tabular}{|c|c|c|c|c|}
\hline & Ingreso & T. esp. & Util rec. & C. perd. \\
\hline Criterios & 0,32258 & 0,25806 & 0,22580 & 0,19354 \\
\hline Alternativa1 & $\mathrm{MB}$ & $\mathrm{B}$ & $\mathrm{MB}$ & $\mathrm{R}$ \\
\hline Alternativa2 & $\mathrm{B}$ & $\mathrm{B}$ & $\mathrm{MB}$ & $\mathrm{M}$ \\
\hline
\end{tabular}

Fuente: Elaboración Propia 

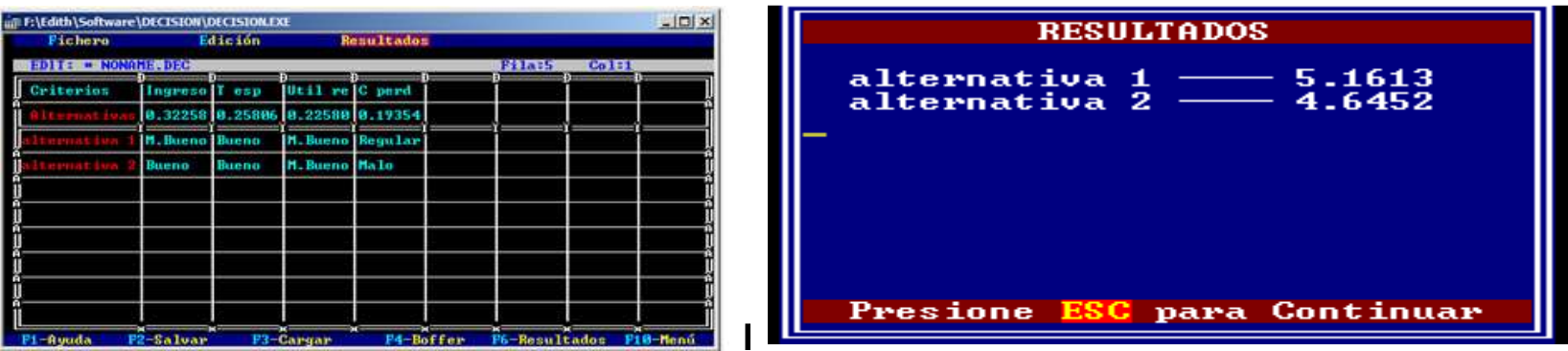

Figura № 10. Algunas salidas del sistema informático

Fuente: Software DECISION

A partir de los resultados obtenidos, se puede plantear que la aplicación de la Simulación de eventos discretos y el enfoque multiatributo de los casos de estudio, ha permitido evaluar cuantitativa y cualitativamente distintas variantes de layout, obteniendo información que permite conocer el comportamiento de cada uno de los sistemas simulados. Esto a su vez constituye información para que el decisor realice la elección final del layout según sus conveniencias y preferencias.

En los casos estudiados se puede apreciar cómo impacta el layout en las variables de salida de interés, tanto aquellas que caracterizan al sistema productivo, como a otras variables relativas a la distribución en planta. En el primer caso, en el que se analiza el comportamiento de los procesos de producción, envase y embalaje de botellas de cervezas y refrescos, se llega a un layout con mejores resultados en cuanto a los retrocesos, posibilitando una disminución en el tiempo de ciclo y un aumento de la producción de bebidas. Mientras que en el segundo caso, donde se simula la instalación que brinda un servicio gastronómico en un nuevo restaurante, la distribución cumple con todos los requisitos solicitados, al mismo tiempo que ofrece a los comensales una visión agradable y contiene la mayor cantidad de mesas que permitirán maximizar los ingresos.

\section{CONCLUSION}

La adaptación del enfoque híbrido de la Simulación y técnicas multicriterio a la evaluación de la distribución en planta de una instalación de producción y(o) servicio permite llegar a resultados que argumentan en mayor medida el diseño a seguir. Por una parte, se aprovechan las ventajas de la Simulación, la que experimentando distintas configuraciones de layout logra la obtención de las variables propias que han sido definidas en esta técnica, mientras que por otra parte, con el empleo de una técnica multiatributo se considera el carácter subjetivo siempre presente en la toma de decisiones y criterios cualitativos, siendo posible

\footnotetext{
"Visión de Futuro" Año 16, Volumen N 23 N², Julio - Diciembre 2019 - Pág. 294 - 311

URL de la Revista: http://visiondefuturo.fce.unam.edu.ar/index.php/visiondefuturo/index

URL del Documento: http://visiondefuturo.fce.unam.edu.ar/index.php/visiondefuturo/issue/view/16

ISSN 1668 - 8708 - Versión en Línea

E-mail: revistacientifica@fce.unam.edu.ar
} 
considerar el conjunto total de atributos de interés para llegar a la mejor decisión según las preferencias del decisor.

Aunque en los casos de estudio que se exponen se aplican como métodos multiatributo el Lexicográfico y el EDIPO, otros son válidos y pueden ser empleados en el enfoque híbrido se propone.

\section{REFERENCIAS}

Acuña-A, et al, (2002). Evaluación de alternativas de distribución de planta por medio de simulación. Revista Tecnología en Marcha. Volumen 15 (ํ1), pág. 44-59.

Aragonés, P. (2010). Técnicas de ayuda a la toma de decisiones en proyectos, Informe Técnico, Universidad Politécnica de Valencia., Valencia, España.

Berumeny, S. y Llamazares, F. (2007). La utilidad de los métodos de decisión multicriterio (como el AHP) en un entorno de competitividad creciente. Revista Cuaderno Administración. Vol. 20, pp. 65-87.

Chase, R.B., y Jacobs, F.R. (2013). Administración de Operaciones. Producción y Cadenas de suministro. Ciudad de México, México. McGraw-Hill.

Fernández, E. (1999). El Método EDIPO para la Ayuda a la Decisión Multicriterio. UPIICSA Tecnología. Ciencia. Cultura., Volumen 3 (N¹9), pp. 25-30.

Fernández, et. al, (2010). An outranking-based fuzzy logic model for collaborative group preferences. TOP., Volumen No18, pp. 444-464.

Fernández, G. y Escribano, M. (2002). Estudio comparativo de Métodos de Ayuda a la Decisión Multicriterio en la valoración y selección de alternativas de inversión. $X$ Jornadas de la Asociación Española de Profesores Universitarios de Matemáticas para la Economía y la Empresa. A.S.E.P.U.M.A., Madrid, España.

Fernández, G. y Escribano, M.C. (07 al 09 de Julio de 2011). La Ayuda a la Decisión Multicriterio: orígenes, evolución y situación actual. VI Congreso Internacional de Historia de la Estadística y de la Probabilidad. Congreso llevado a cabo en Centro Asociado de la UNED de Valencia Francisco Tomás y Valiente. Valencia, España.

Garza, R., González, C. y Pérez, I. (2012). Comparación de métodos de ayuda a la decisión multiatributo en la solución de problemas de itinerarios de vehículos. Revista Investigación Operacional, Volumen No33 (N2), pp. 152 - 159.

González, et. al, (2014). Enfoque hibrido simulación - proceso analítico jerárquico: caso de estudio del rediseño de un restaurante. Revista Métodos Cuantitativos para la Economía y la Empresa, Volumen $\mathrm{N}^{\circ} 17$, pp $23-41$.

Hillier, F. and Lieberman, G. (2015). Introducción a la Investigación de Operaciones, 10ma edición. Ciudad de Mexico, México. McGraw-Hill Interamericana. 
Kelton, WD. et al. (2009). Simulation with ARENA, 3ra edición. Ciudad y país. Editorial McGraw Hill Science Engineering.

Lara, L. (2016).Teoría semántica y método lexicográfico, 1ra edición. México. Editorial El Colegio de México.

Marcoux et al, (2005). Models and Methods for Facilities Layout Design from an Applicability to Real-World Perspective. New York, Springer US, Volumen, pp. 123-170.

Martínez, et al, (2018). Integración de la Simulación, la Regresión y la Optimización Multiobjetivo para determinar los recursos en un banco. Revista Investigación Operacional. Vol. 39 (No1), pp. 140-150.

Mejia, H. y Galofre, M. (2008). Aplicación de software de simulación como herramienta en el rediseño de plantas de producción en empresas del sector de alimentos. Revista Prospectiva, Vol. 6 (N2) pp. 39-45.

Panwar D., Patra S. (2017). Localization in Fast Food industry: A case study on McDonald's strategy in India. Journal of Arts, Science \& Commerce, Vol. VIII, (No1), pp. 7074.

Pochamarn, et al, (2008). Using Promodel as a simulation tool to assist plant layout design and planning: Case study plastic packaging factory. Songklanakarin journal of Sciencie and Technology (SJTS), Vol. 30. pp. $117-123$.

Rodríguez, et al, (2002). Utilización de la simulación para el rediseño de la sección de expedición de una PYME (Aux. Sector Calzado). Il Conferencia de Ingeniería de Organización. Conferencia llevada a cabo en Vigo, España.

Saaty, T. and Vargas, L. (2012). Models, Methods, Concepts \& Applications of the Analytic Hierarchy Process, 2da edición. Ciudad y país. Springer US.

Soto de la Vega, et al, (2014). Metodología para localización de centros de distribución a través de análisis multicriterio y optimización. Revista Dyna, Volumen 81 (No184), pp . 2835.

Suárez- Sánchez, L. (2017). Procedimiento para la conformación de un layout con el empleo de la simulación (Trabajo de Diploma para optar por el título de Ingeniero Industrial), Universidad Tecnológica de la Habana José A. Echeverría, Facultad de Ingeniería Industrial. La Habana, Cuba.

Tavella, et al, (2014). Estudio Comparativo de Métodos Multicriterio para el análisis de la Localización Sustentable de Parques Industriales Regionales. Revista Facultad de Ciencias Exactas, Físicas y Naturales, Vol. 1 (№1), pp $41-48$.

Wiendahl, et al, (2015). Handbook Factory Planning and Design. Berlin Heidelberg. Springer-Verlag.

\footnotetext{
"Visión de Futuro" Año 16, Volumen No 23 N², Julio - Diciembre 2019 - Pág. 294 - 311

URL de la Revista: http://visiondefuturo.fce.unam.edu.ar/index.php/visiondefuturo/index

URL del Documento: http://visiondefuturo.fce.unam.edu.ar/index.php/visiondefuturo/issue/view/16

ISSN 1668 - 8708 - Versión en Línea 


\section{RESUMEN BIOGRAFICO}

\section{Rosario Garza Ríos}

Doctora en Ciencias Técnicas obtenido en el Instituto Superior Politécnico José Antonio Echeverría, actual Universidad Tecnológica de La Habana donde se desempeña como Profesor Titular. Jefa de la disciplina de Estadística e Investigación de Operaciones. Investiga en Simulación, Uso de técnicas cuantitativas para la toma de Decisiones y Enfoque Multicriterio.

\section{Edith Martínez Delgado}

Doctora en Ciencias Técnicas, obtenido en el Instituto Superior Politécnico José Antonio Echeverría, actual Universidad Tecnológica de La Habana, donde se desempeña como Profesor Titular. Presidenta del tribunal de categorización docente. Investiga en Distribución en Planta, Uso de técnicas cuantitativas para la toma de Decisiones, Logística, y enfoque híbrido Multicriterio-Simulación. 